\title{
The role of social support, detachment, and depressive and anxiety symptoms in suicidal thoughts and behaviours during the Covid-19 lockdown: Potential pathways
}

\author{
Aina Gabarrell-Pascuet ${ }^{1,2}$ (D) Mireia Félez-Nóbrega ${ }^{1,2}$ • Paula Cristóbal-Narváez ${ }^{1,2} \cdot$ Philippe Mortier $^{3,4}$. \\ Gemma Vilagut $^{3,4} \cdot$ Beatriz Olaya $^{1,2}$ • Jordi Alonso ${ }^{3,4,5} \cdot$ Josep Maria Haro ${ }^{1,2,6,7} \cdot$ Joan Domènech-Abella ${ }^{1,2,8}$
}

Accepted: 5 August 2021

(C) The Author(s), under exclusive licence to Springer Science+Business Media, LLC, part of Springer Nature 2021

\begin{abstract}
During the COVID-19 pandemic, anxiety and depressive symptoms, as well as problems related to social relationships, such as available social support and feelings of detachment from others, have worsened. These factors are strongly associated with suicidal thoughts and behaviours (STB). The effects of feelings of detachment on mental health and on STB have been scarcely studied, together with the relation that it may have with available social support. Therefore, the aim of the present study was to assess potential pathways connecting these conditions. A nationally representative sample of Spanish adults $(N=3305)$ was interviewed during the COVID-19 pandemic (June 2020). STB, social support, and depressive and anxiety symptoms were measured with the C-SSRS (modified version), OSSS-3, PHQ-8, and GAD-7 scales, respectively. Multivariable logistic regression models and mediation analyses were performed. Social support and some of its components (i.e., social network size and relations of reciprocity) were associated with lower odds of STB. Detachment significantly mediated (22\% to $25 \%)$ these associations. Symptoms of emotional disorders significantly mediated the association between social support components ( $29 \%$ to $38 \%$ ) - but not neighbourhood support - with STB, as well as the association between detachment and higher odds of STB (47\% to 57\%). In both cases, depressive symptoms were slightly stronger mediating factors when compared to anxiety symptoms. Our findings suggest that interventions aimed at lowering depressive and anxiety symptoms, and STB should provide social support and help tackle the feeling of detachment in a complementary way.
\end{abstract}

Keywords Suicide $\cdot$ Detachment $\cdot$ Social support $\cdot$ Anxiety symptoms $\cdot$ Depressive symptoms $\cdot$ COVID-19

\section{Introduction}

Severe Acute Respiratory Syndrome Coronavirus 2 (SARSCoV-2) was first detected in December 2019 in Wuhan, China, in several patients diagnosed with viral pneumonia

Aina Gabarrell-Pascuet

aina.gabarrell@sjd.es

1 Teaching, Research \& Innovation Unit, Parc Sanitari Sant Joan de Déu, C/ Doctor Antoni Pujades, 42, ES-08830 Sant Boi de Llobregat, Barcelona, Spain

2 Centre for Biomedical Research on Mental Health (CIBERSAM), Instituto de Salud Carlos III, Madrid, Spain

3 Health Services Research Unit, IMIM (Hospital del Mar Medical Research Institute), Barcelona, Spain
(Yousefi et al., 2020). SARS-CoV-2 causes the Coronavirus disease (COVID-19), which has caused about 142.6 M confirmed cases and 3.0 M deaths worldwide in approximately 1 year, and from which 77,220 deaths have occurred in Spain (WHO, 2020). Due to the lack of specific medical treatment,

4 Centro de Investigación Biomédica en Red de Epidemiología y Salud Pública (CIBERESP), Instituto de Salud Carlos III, Madrid, Spain

5 Department of Experimental and Health Sciences, Pompeu Fabra University (UPF), Barcelona, Spain

6 Instituto de Investigación Sanitaria Princesa (IP), Madrid, Spain

7 Department of Medicine, Universitat de Barcelona (UB), Barcelona, Spain

8 Department of Sociology, Universitat de Barcelona (UB), Barcelona, Spain 
the main measures to contain COVID-19's spread and flatten its infection curve have been based on physical distancing, contact clustering in social bubbles, and stay-at-home orders. Specifically, in Spain, lockdown restrictions started on 14 March with 1811 confirmed cases and 59 deaths that day, and peaked on 29 March with 13,749 confirmed cases and 909 deaths (WHO, 2020). By the end of the lockdown period (21 June), when the number of cases had already stabilized, Spain had the eighth highest number of cases worldwide (i.e. 249,659), and the fifth highest COVID-19 death rate (i.e. 60.7/ 100,000 ) in the world (Ritchie et al., 2020). The quarantine measures of self-isolation may have affected the social relationships of those whose only social contact was out of the home. These restriction measures have also broadened a range of mental health problems associated with the COVID-19 pandemic, including symptoms of anxiety and depressive disorders, posttraumatic stress disorder (PTSD), loneliness, stress, and psychological distress in the general population (Chen et al., 2021; Li et al., 2020; Vindegaard \& Benros, 2020; Xiong et al., 2020).

For the time being, the specific effect of the COVID-19 pandemic on worldwide suicide mortality remains unclear (John et al., 2020; Tanaka \& Okamoto, 2021). However, the effects of the pandemic on mental health and social connectedness, as well as on socioeconomic conditions, may include potentially increased suicide rates during and after the pandemic (Banerjee et al., 2021; Sher, 2020a; Thakur \& Jain, 2020; Xiong et al., 2020). Therefore, it is necessary to be prepared and to consider preventive measures to mitigate suicide risk associated with the COVID-19 pandemic (Gunnell et al., 2020). In this regard, understanding the risk factors for suicide and their possible combinations becomes essential.

Nearly 800,000 people die by suicide every year and estimates suggest that for each suicide there are 20 suicide attempts (WHO, 2019). In 2016, 53,500 deaths by suicide occurred in the European Union (Eurostat, 2021), corresponding to $1 \%$ of all deaths. Around three quarters of these deaths by suicide were among men, while more than half of all suicides were committed at age 50 or over. In the Spanish general population, estimates of lifetime prevalence of suicidal thoughts and suicidal behaviours in 2014 were $3.67 \%$ and $1.46 \%$, respectively (Miret et al., 2014). Although Spain has traditionally shown some of the lowest suicide rates in Europe (Alfonso-Sánchez et al., 2020), spikes in the rates of suicidal thoughts and behaviours (STB) have been reported worldwide in previous viral disease outbreaks (Banerjee et al., 2021; Leaune et al., 2020).

The effects of social conditions on STB are well documented: socioeconomic crises and low levels of social protection (i.e., lower expenditure that supports families, active labour market programmes, rent subsidies, medical services, etc.) increase the risk of suicide, particularly among those with greater socioeconomic and educational disadvantages
(Lorant et al., 2018; Stuckler et al., 2009). Moreover, objective and subjective components of social relationships may also affect the likelihood of suffering STB in a complementary way (Calati et al., 2019; Van Orden et al., 2010). Objective factors include having quantifiable contact with, or support from, other people, while subjective factors include positive or negative feelings that could be associated with those objective factors. For the present study, we have used social support (a parameter comprising social network size, relations of reciprocity based on the sense of concern from other people, and neighbourhood support) and partner status as objective factors; and feelings of detachment from others as a subjective factor, which have been described as a key construct predicting suicide (Durkheim, 1951).

Detachment or estrangement from others has also been identified as an intrusion symptom associated with traumatic events, and it is a common PTSD symptom (American Psychiatric Association, 2013). PTSD and its symptoms - as already observed during and after the SARS pandemic of 2003 - are likely to increase in the long-term due to the COVID-19 pandemic, particularly among the most vulnerable groups and people at increased risk for severe illness (e.g., COVID-19 patients and their close contacts, health care workers and other hospital staff, persons with a psychiatric illness history or with underlying health conditions, older people, individuals who reside in high COVID-19 prevalence areas, etc.) (Brooks et al., 2020; Bryant-Genevier et al., 2021; Mazza, De Lorenzo, et al., 2020b; Sher, 2020b; Vindegaard \& Benros, 2020). In individuals with high rates of trauma exposure (Hyatt et al., 2020), and during the COVID-19 pandemic (Mazza, Ricci, et al., 2020a), detachment has been positively associated with symptoms of emotional disorders (i.e., anxiety and depressive disorders). This could contribute to explaining the growing evidence of a rise in symptoms of emotional disorders during the COVID-19 outbreak (Ettman et al., 2020; Li et al., 2020). Moreover, emotional disorders have been found to increase the risk of suicide (Hawton et al., 2013; Too et al., 2019) and to be affected by objective and subjective factors of social relationships (Liu et al., 2020; Santini et al., 2015).

The fact that the few studies that have studied detachment have related it to emotional disorders, which in turn have increased during the COVID-19 pandemic, and are a risk factor for suicide, leads us to want to know more about the effects of detachment in these mental health problems. On the other hand, social support is a protective factor for both emotional disorders and suicide, and we wondered what role detachment plays in these relationships. Therefore, we hypothesized that detachment could partially explain the relationship between having a lower social support and living without a partner, with higher odds of having STB; and concurrently, we hypothesized that the relationship amidst these variables with STB could be mediated by symptoms of emotional disorders. 
As far as we aware, scarce research has inquired these potential mediating pathways, and, in particular, it has seldom been examined in large epidemiological cohorts of general population. So, an appropriate first step is to investigate the relationships between these constructs cross-sectionally. Hence, the aims of the present study are, firstly, to explore the association among social support, its components (i.e., social network size, relations of reciprocity, and neighbourhood support), and partner status with STB; and to study the extent to which detachment might explain this association (hypothetical model A, Fig. 1a). Secondly, we aim to research whether - and to what degree - symptoms of depressive and anxiety disorders mediate (i.e., explain) the associations among detachment, social support and its components, and partner status with STB (hypothetical model B, Fig. 1b).

\section{Methods}

\section{Study Design}

This study was part of the MIND-COVID project (MIND/COVID, 2020), for which data were collected from a cross-sectional survey conducted in a random and representative sample of the Spanish general adult population. The inclusion criteria were (1) being 18 years or older and not institutionalized, (2) being a resident in Spain at the beginning of the study, and (3) having access to either a mobile or a landline telephone. The exclusion criteria were (1) having a language barrier to understand the survey and (2) not giving explicit consent to participate.

Trained professional interviewers administered the survey with computer-assisted telephone interviews between the 1st and the 30th of June 2020, in order to obtain data from the last period of the lockdown. A dual-frame random digit dialling (DFRDD) telephone survey was used to create the sample. The most recent data indicate that in 2019 in Spain $98.5 \%$ of households had a mobile phone and $74.9 \%$ had a landline telephone (INE, 2020), which is why the DFRDD included more mobile than landline telephone numbers. First, a sample of Spanish mobile telephone numbers was generated through an automated system. Subsequently, landline numbers were selected from an internal database developed and maintained by the survey company to ensure that all Spanish geographical areas were adequately represented. Up to seven calls at different times of the day were attempted to each number. The distribution of the interviews was planned according to proportional quotas of the Spanish population in terms of age group, sex, and region of residence.

A total of 138,656 numbers were sampled, with a final split of $71 \%$ mobile and $29 \%$ landline telephones. Of these, 45,002 were non-eligible (i.e. non-existing numbers $[43,120]$, numbers of enterprises [984], numbers of persons with Spanish language barriers [444], fax numbers [268], and numbers belonging to quota that were already completed [186]) and 72,428 had unknown eligibility (i.e. no contact was made after
Fig. 1 Hypothetical mediation models of suicidal thoughts and behaviours (STB): (a) mediated by detachment, and (b) mediated by emotional disorders symptoms. ${ }^{\text {a }}$ Mediation percentages of the association of each independent variable with $\mathrm{STB}$, in the same order as the listed independent variables. n.s. $=$ non-statistically significant

\section{INDEPENDENT VARIABLES}

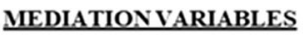

DEPENDENT VARIABLE

(a)

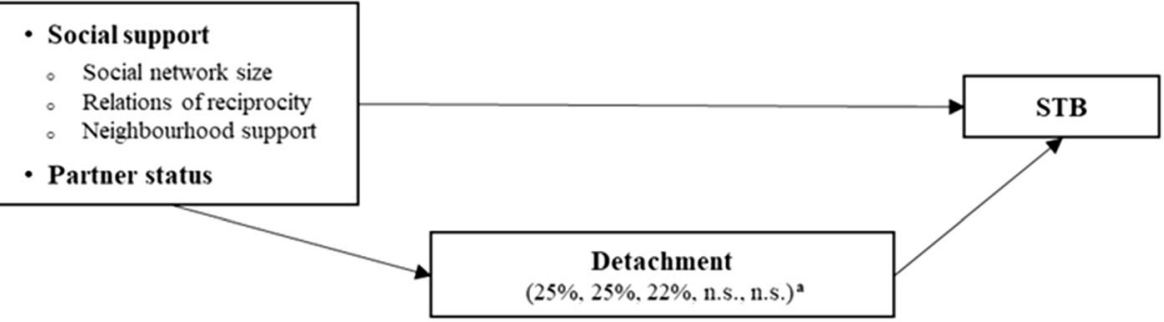

(b)

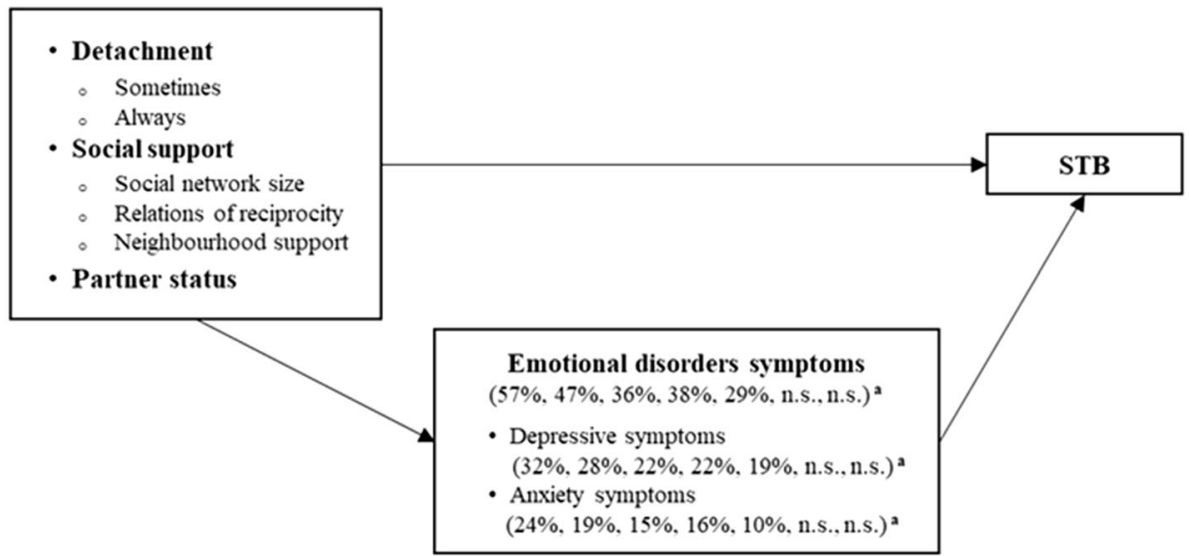


the seven attempted calls), resulting in a cooperation rate (i.e., the proportion of all cases interviewed of all eligible units ever contacted) of $16.5 \%$.

A total of 3500 individuals responded to the interview. For the present study, 195 individuals were excluded, since no answers regarding their feelings of detachment in the context of lockdown confinement were obtained. A total sample of 3305 individuals was included in the final analyses.

\section{Ethics Statement}

Ethical approval from the relevant ethical committees (Parc Sanitari Sant Joan de Déu, Barcelona, Spain (PIC 86-20) and Parc de Salut Mar Clinical Research Ethics Committee (protocol 2020/9203/I)) was obtained. Participants were thoroughly informed about the objectives and procedures of the study. Each respondent had to provide oral consent prior to participation.

\section{Measures}

\section{Study Variables}

A modified self-report version of selected items from the Columbia Suicide Severity Rating Scale (C-SSRS) was used to determine whether the participants had any suicidal thoughts and behaviours in the prior 30 days (Posner et al., 2011). It consists of dichotomous (yes/no) items that assess passive suicidal ideation ("wish you were dead or would go to sleep and never wake up"), active suicidal ideation ("have thoughts of killing yourself"), suicide plans ("think about how you might kill yourself [e.g., taking pills, shooting yourself] or work out a plan of how to kill yourself"), and suicide attempts ("make a suicide attempt [i.e., purposefully hurt yourself with at least some intent to die]).

Detachment from others was determined with a single-item question. Participants were given a list of experiences they may have had during the lockdown ordered by the Spanish government (March 14 to June 21, 2020), and they had to indicate how often they had them. One of the listed experiences was "feeling detached" and the possible answers were "never", "sometimes", "seldom", “often", or "always". For the analyses, detachment frequency was divided into three groups: never, sometimes (comprising sometimes and seldom), and always (comprising often and always).

Depressive symptoms were measured with the Patient Health Questionnaire Depression Scale (PHQ-8), which is Likert-type scale containing 8 items ranging from 0 to 3 (Kroenke et al., 2009). The total score was obtained by adding the responses and ranging them from 0 to 24. The 7-item Generalized Anxiety Disorder Scale (GAD-7) was included to measure anxiety symptoms (Newman et al., 2002). It is a 7item measure, with items scored from 0 to 3 , and a total score ranging from 0 to 21 . In both scales, higher values represent greater emotional disorders symptoms.

The components of social support were assessed using the Oslo Social Support Scale (OSSS-3), which includes questions about social network size, relations of reciprocity, and neighbourhood support (Kocalevent et al., 2018). These questions were, respectively, "How many people are so close to you that you can count on them if you have great personal problems?" (4 "more than 5", 3 "from 3 to 5", 2 "from 1 to 2", 1 "none"); "How much interest and concern do people show in what you do?" (5 "a lot", 4 "some", 3 "uncertain", 2 "little", 1 "none"), and "How easy is it to get practical help from neighbours if you should need it?" (5 "very easy", 4 "easy", 3 "possible", 2 "difficult", 1 "very difficult"). In the current study, social network size, relations of reciprocity, and neighbourhood support were used as interest variables individually and assembled together to determine the social support score. The total social support score was obtained by adding the responses and ranging them from 3 to 14, with higher values representing stronger levels of social support. Moreover, partner status was also covered by asking participants to indicate whether or not they lived with an intimate partner.

\section{Covariates (Sociodemographic and Health Measures)}

Sociodemographic factors and health measures potentially related to the study variables were included as confounding variables in the adjusted models.

Sociodemographic factors included age, sex, and educational level. Age was categorized into five groups: 1834 years, 35-49 years, 50-64 years, 65-79 years, and 80 years or over. Categories for the highest level of education obtained were primary (primary education or less), lower-secondary (secondary education or high school), higher-secondary (vocational education and training, Spanish baccalaureate), and post-secondary (higher education including advanced technician and university education or higher).

Health measures included respondents' health perception regarding pain and malaise and their ability to carry on daily activities, and number of physical health problems. Categories for pain/malaise were none-mild, moderate, and severe-extreme. The inability to perform daily activities (i.e., working, studying, doing household chores, leisure activities, and family activities) was categorized as none (no difficulty doing daily activities), mild-moderate, and severe-extreme (severe problems doing daily activities or complete inability to perform them).

Physical health problems included respiratory diseases, cardiovascular problems, diabetes, cancer, chronic liver disease, immunological problems, and others. This question was categorized according to the amount of physical health problems as none, one, and two or more. 
The existence of pre-pandemic lifetime mental disorders was assessed using a checklist based on the Composite International Diagnostic Interview (CIDI) that screens for depression, bipolar disorder, anxiety, panic attacks, alcohol and drug use problems, and "other" mental disorders (Kessler \& Üstün, 2004).

Finally, a history of COVID-19 infection (positive test and/ or medical diagnosis) and/or having been in isolation or quarantine related to COVID-19 was also considered through a dichotomous variable indicating the absence or presence of at least one of these conditions.

\section{Statistical Analysis}

In order to compensate for survey non-response and increase the representativeness of the sample, post-stratification weighting was used to restore the distribution of the Spanish general adult population according to age, sex, and geographic area. The median value of individuals with missing values across the analysed variables was less than $1 \%$, and all variables had less than $5 \%$ of missing data, which was addressed using single multivariate imputation with chained equations (van Buuren, 2018).

Descriptive analyses of the study sample included frequencies and weighted proportions for categorical variables and mean with standard deviation for continuous variables.

Unadjusted and adjusted logistic regression models were fitted to explore the association between the study variables and covariates with STB in the prior 30 days (dependent variable). Those variables that predicted the outcome $(p<0.20)$ (Mickey \& Greenland, 1989) in the unadjusted models were introduced as control variables in the adjusted models and mediation analyses. Odds ratio (95\% confidence interval) and significance when $p<0.05$ were reported for each model.

Figure 1 illustrates the cross-sectional hypothetical models (A and B) to analyse the possible mediation effects on STB (dependent variable). Model A included social support components and partner status as independent variables, while detachment was considered a mediation variable. Model B included detachment, social support (and its components), and partner status as independent variables, and symptoms of emotional disorders as mediation variables. Analyses were performed using the Karlson-Holm-Breen (KHB) method (Breen et al., 2013), which breaks down the total effect of a variable into a direct and an indirect (i.e., mediational) effect. All mediation analyses included the following confounding variables: sex, age, education, malaise/pain, inability to perform daily activities, physical health problems, pre-pandemic mental disorders, history of COVID-19 infection/quarantine, and, when applicable, social support and partner status.

To obtain a more detailed understanding of the mediational effects of anxiety and depressive symptoms, the "disentangle" option was used in hypothetical model B. In so doing, a representation of how much of the difference between the total and the indirect effect is contributed by each emotional disorder was obtained. The level of statistical significance was set at $p<0.05$ (2-sided). The percentages of mediation were only considered in the presence of a significant indirect effect.

Stata version SE 13 was used to perform all the analyses.

\section{Results}

Table 1 shows the characteristics of the study sample $(N=$ 3305 ), which consisted of a nearly balanced proportion of females and males. The percentage of participants in each age group ranged from $20 \%$ to $30 \%$, except for those aged over $80(3.5 \%)$. Roughly half of the participants had a postsecondary education level (49\%), whereas about $8 \%$ had a primary education level. Most participants did not have malaise or pain (85\%), inability to perform daily activities $(91 \%)$, or any physical health problems $(60 \%)$. Approximately one third of the sample (34\%) had pre-pandemic lifetime mental disorders and just $1 \%$ of the sample had a history of COVID19 infection or quarantine. Overall, almost $25 \%$ of participants reported at least some evidence of detachment during the lockdown, with $5 \%$ reporting having always felt detached. $4.5 \%$ of the study sample had had STB in the previous 30 days, of whom $2.75 \%$ had passive suicidal ideation only, while the other $1.79 \%$ had an active suicidal ideation, plan, or attempt. The characteristics significantly more frequent among individuals with STB were being female, having a lower level of education, feeling pain or malaise, being unable to perform daily activities, having one or more physical health problems, and having pre-pandemic lifetime mental disorders. Not living with a partner, having lower levels of social support - including a smaller social network, fewer relations of reciprocity, and less neighbourhood support -, feeling detached, and having higher levels of anxiety and depressive symptoms were also significantly associated with STB.

Table 2 reports odds for STB. The most frequent characteristics of individuals with STB on Table 1 were also significantly associated with higher odds for STB in the unadjusted model (Model 1); whereas in the adjusted model (Model 2), higher levels of depressive and anxiety symptoms, always feeling detachment, severe/extreme malaise/pain, and having pre-pandemic lifetime mental disorders remained significantly related to higher odds for STB.

As Table 3 shows, social support was significantly related to lower STB, and detachment significantly mediated this association with a percentage of mediation of $24.6 \%$. Specifically, the components of social support significantly related to lower STB were social network size and sense of reciprocity, with detachment explaining $25.3 \%$ and $22.2 \%$ of these relationships, respectively. 
Table 1 Characteristics of the study sample, of individuals without any suicidal thoughts and behaviours (STB), and of individuals with any STB

\begin{tabular}{|c|c|c|c|c|}
\hline Characteristic & Total sample $(N=3305)$ & Individuals without STB $(n=3155)$ & Individuals with STB $(n=150)$ & $p$-value ${ }^{\mathrm{a}}$ \\
\hline \multicolumn{5}{|l|}{ Sex, $n(\%)$} \\
\hline Male & $1462(48.69)$ & $1416(96.71)$ & $46(3.29)$ & \multirow[t]{2}{*}{$p<0.01$} \\
\hline Female & $1843(51.31)$ & $1739(94.27)$ & $104(5.73)$ & \\
\hline \multicolumn{5}{|l|}{ Age, $n(\%)$} \\
\hline $18-34$ & $661(22.12)$ & $626(94.72)$ & $35(5.28)$ & \multirow[t]{5}{*}{0.391} \\
\hline $35-49$ & $988(28.12)$ & 947 (95.95) & $41(4.05)$ & \\
\hline $50-64$ & $1053(25.52)$ & $1009(96.02)$ & $44(3.98)$ & \\
\hline $65-79$ & $517(20.77)$ & $494(95.52)$ & $23(4.48)$ & \\
\hline $80+$ & $86(3.47)$ & $79(91.75)$ & $7(8.25)$ & \\
\hline \multicolumn{5}{|l|}{ Education, $n(\%)$} \\
\hline Primary & $228(7.86)$ & $204(89.18)$ & $24(10.82)$ & \multirow[t]{4}{*}{$p<0.001$} \\
\hline Lower-secondary & $1131(34.38)$ & $1067(94.72)$ & $64(5.28)$ & \\
\hline Higher-secondary & $283(8.49)$ & $270(94.89)$ & $13(5.11)$ & \\
\hline Tertiary & $1663(49.27)$ & $1614(97.08)$ & $49(2.92)$ & \\
\hline \multicolumn{5}{|l|}{ Malaise/pain, $n(\%)$} \\
\hline None-mild & $2800(84.59)$ & $2711(96.81)$ & $89(3.19)$ & \multirow[t]{3}{*}{$p<0.001$} \\
\hline Moderate & $358(10.93)$ & $325(91.14)$ & $33(8.86)$ & \\
\hline Severe-extreme & $147(4.48)$ & $119(80.49)$ & $28(19.51)$ & \\
\hline \multicolumn{5}{|l|}{ Inability to perform daily activities, $n(\%)$} \\
\hline None & $3013(91.05)$ & $2903(96.32)$ & $110(3.68)$ & \multirow[t]{3}{*}{$p<0.001$} \\
\hline Mild-moderate & $252(7.71)$ & $223(89.20)$ & $29(10.80)$ & \\
\hline Severe-extreme & $40(1.24)$ & $29(71.41)$ & $11(28.59)$ & \\
\hline \multicolumn{5}{|l|}{ Physical health problems, $\mathrm{n}(\%)$} \\
\hline None & $2006(60.37)$ & $1932(96.44)$ & $74(3.56)$ & \multirow[t]{3}{*}{$p<0.001$} \\
\hline One & $940(28.55)$ & $901(95.67)$ & $39(4.33)$ & \\
\hline Two or more & $359(11.08)$ & $322(89.62)$ & $37(10.38)$ & \\
\hline \multicolumn{5}{|l|}{ Pre-pandemic mental disorder, $n(\%)$} \\
\hline No & $2152(65.77)$ & $1040(98.23)$ & $113(1.77)$ & \multirow[t]{2}{*}{$p<0.001$} \\
\hline Yes & $1153(34.23)$ & $2115(90.15)$ & $37(9.85)$ & \\
\hline \multicolumn{5}{|l|}{ COVID-19 infection/quarantine, $n(\%)$} \\
\hline No & $3270(98.94)$ & 3121 (95.44) & $149(4.56)$ & \multirow[t]{2}{*}{0.631} \\
\hline Yes & $35(1.06)$ & $34(97.28)$ & $1(2.72)$ & \\
\hline \multicolumn{5}{|l|}{ Living with a partner, $n(\%)$} \\
\hline No & $1168(37.25)$ & $1093(93.66)$ & $75(6.34)$ & \multirow[t]{2}{*}{$p<0.001$} \\
\hline Yes & $2137(62.75)$ & $2062(96.53)$ & $75(3.47)$ & \\
\hline Social support scale (3-14), mean(SD) & $11.11(1.89)$ & $11.12(1.84)$ & $9.99(2.59)$ & $p<0.001$ \\
\hline Social network size $(1-4)$, mean(SD) & $3.42(0.69)$ & $3.44(0.67)$ & $3.11(0.90)$ & $p<0.001$ \\
\hline Relations of reciprocity (1-5), mean(SD) & $4.16(0.87)$ & $4.19(0.84)$ & $3.66(1.23)$ & $p<0.001$ \\
\hline Neighbourhood support (1-5), mean(SD) & $3.53(1.08)$ & $3.54(1.07)$ & $3.21(1.27)$ & $p<0.001$ \\
\hline Depressive symptoms $(0-24)$, mean(SD) & $3.96(4.58)$ & $3.62(4.17)$ & $11.04(6.63)$ & $p<0.001$ \\
\hline Anxiety symptoms $(0-21)$, mean $(\mathrm{SD})$ & $3.64(4.29)$ & $3.34(3.95)$ & $10.11(5.92)$ & $p<0.001$ \\
\hline \multicolumn{5}{|l|}{ Detachment, $n(\%)$} \\
\hline Never & $2479(75.33)$ & $2423(97.70)$ & $56(2.30)$ & \multirow[t]{3}{*}{$p<0.001$} \\
\hline Sometimes & $653(19.53)$ & $606(92.93)$ & $47(7.07)$ & \\
\hline Always & $173(5.14)$ & $126(72.30)$ & $47(27.70)$ & \\
\hline \multicolumn{5}{|l|}{$\mathrm{STB}^{\mathrm{b}}, n(\%)$} \\
\hline None & 3155 (95.46) & - & - & \\
\hline Passive suicidal ideation & $91(2.75)$ & - & - & \\
\hline Active suicidal ideation, plan, or attempt & $59(1.79)$ & - & - & \\
\hline
\end{tabular}

Frequencies and weighted proportions (in percentages) are displayed for categorical variables, and unweighted means with standard deviation (SD) for continuous variables

${ }^{a}$ The difference between the values for individuals with and without any STB was tested with Chi-squared tests and t tests for categorical and continuous variables, respectively

${ }^{\mathrm{b}}$ STB refers to having had suicidal thoughts and behaviours in the past 30 days, and includes passive suicidal ideation, active suicidal ideation, suicide plans, and suicide attempts

As displayed in Table 4, feeling detachment was significantly related to higher STB (compared to never feeling detachment). Symptoms of emotional disorders explained $56.6 \%$ and $47.3 \%$ of the association between feeling detachment sometimes and always, respectively. In both categories of detachment (sometimes and always), depressive symptoms were responsible for around $30 \%$ of the whole emotional disorder mediation, with the percentages being 
Table 2 Association of study covariates with suicidal thoughts and behaviours (STB)

\begin{tabular}{|c|c|c|}
\hline & Bivariate Model (1) & Multivariate Model (2) \\
\hline Social support scale (3-14) & $0.79(0.72,0.86)^{* * *}$ & $0.93(0.84,1.02)$ \\
\hline Depressive symptoms $(0-24)$ & $1.24(1.20,1.27)^{* * *}$ & $1.10(1.05,1.16)^{* * *}$ \\
\hline Anxiety symptoms (0-21) & $1.25(1.22,1.29)^{* * *}$ & $1.08(1.02,1.14)^{* *}$ \\
\hline \multicolumn{3}{|l|}{ Detachment } \\
\hline Never & Ref. & Ref. \\
\hline Sometimes & $3.22(2.14,4.86) * * *$ & $1.38(0.83,2.27)$ \\
\hline (Almost) always & $16.25(10.46,25.24)^{* * *}$ & $3.09(1.68,5.67)^{* * *}$ \\
\hline \multicolumn{3}{|l|}{ Sex } \\
\hline Male & Ref. & Ref. \\
\hline Female & $1.79(1.24,2.57)^{* *}$ & $1.13(0.74,1.75)$ \\
\hline \multicolumn{3}{|l|}{ Age } \\
\hline $18-34$ & Ref. & Ref. \\
\hline $35-49$ & $0.76(0.47,1.21)$ & $1.08(0.63,1.86)$ \\
\hline $50-64$ & $0.74(0.47,1.18)$ & $0.87(0.48,1.59)$ \\
\hline $65-79$ & $0.84(0.49,1.45)$ & $1.13(0.54,2.25)$ \\
\hline $80+$ & $1.61(0.69,3.77)$ & $1.85(0.53,6.49)$ \\
\hline \multicolumn{3}{|l|}{ Education } \\
\hline Primary & Ref. & Ref. \\
\hline Lower-secondary & $0.46(0.28,0.76)^{* *}$ & $0.55(0.28,1.09)$ \\
\hline Higher-secondary & $0.44(0.22,0.91)^{*}$ & $0.78(0.32,1.89)$ \\
\hline Tertiary & $0.25(0.15,0.42)^{* * *}$ & $0.49(0.24,1.00)$ \\
\hline \multicolumn{3}{|l|}{ Malaise/pain } \\
\hline None-mild & Ref. & Ref. \\
\hline Moderate & $2.95(1.92,4.53) * * *$ & $1.74(0.99,3.06)$ \\
\hline Severe-extreme & $7.37(4.56,11.90)^{* * *}$ & $2.32(1.05,5.10)^{*}$ \\
\hline \multicolumn{3}{|c|}{ Inability to perform daily activities } \\
\hline None & Ref. & Ref. \\
\hline Mild-moderate & $3.17(2.03,4.95)^{* * *}$ & $1.10(0.59,2.04)$ \\
\hline Severe-extreme & $10.48(4.97,22.18)^{* * *}$ & $1.17(0.40,3.49)$ \\
\hline \multicolumn{3}{|l|}{ Physical health problems } \\
\hline None & Ref. & Ref. \\
\hline One & $1.23(0.82,1.84)$ & $0.81(0.48,1.36)$ \\
\hline Two or more & $3.14(2.05,4.81) * * *$ & $1.14(0.60,2.17)$ \\
\hline \multicolumn{3}{|l|}{ Pre-pandemic mental disorder } \\
\hline No & Ref. & Ref. \\
\hline Yes & $6.05(4.10,8.95)^{* * *}$ & $2.35(1.51,3.68)^{* * *}$ \\
\hline \multicolumn{3}{|l|}{ COVID-19 infection/quarantine } \\
\hline No & Ref. & Ref. \\
\hline Yes & $0.59(0.08,4.31)$ & $0.89(0.52,1.20)$ \\
\hline \multicolumn{3}{|l|}{ Living with a partner } \\
\hline No & Ref. & Ref. \\
\hline Yes & $0.53(0.38,0.74)^{* * *}$ & $0.79(0.52,1.20)$ \\
\hline
\end{tabular}

Model 1 represents one bivariate logistic regression model for each of the variables presented in rows. Model 2 are the multivariable logistic regression models (adjusted for all the variables shown in the table). Odds ratios and $95 \%$ confidence intervals are displayed. In both models sampling weights are considered

$* p<0.05 ; * * p<0.01 ; * * p<0.001$
$32.3 \%$ when feeling detachment sometimes and $27.8 \%$ when always feeling detachment. The mediation of anxiety symptoms, on the other hand, was slightly lower in both cases (24.2\% vs. $19.5 \%$ ). 
Table 3 Association (multivariate regression) of social network characteristics and suicidal thoughts and behaviours (STB), with detachment as mediating factor (KHB method)

\begin{tabular}{lll}
\hline Social network characteristics & Coefficient $(95 \% \mathrm{CI})$ & $\%$ Mediated \\
\hline Social support (scale 3-14) & & \\
Total & $-0.16(-0.25,-0.07)^{* *}$ & \\
Direct & $-0.12(-0.22,-0.03)^{*}$ & \\
Indirect (detachment) & $-0.04(-0.06,-0.02)^{* * *}$ & $24.63 \%$ \\
Social network size (1-4) & & \\
Total & $-0.37(-0.62,-0.12)^{* *}$ & \\
Direct & $-0.28(-0.53,-0.02)^{*}$ & \\
Indirect (detachment) & $-0.09(-0.13,-0.05)^{* * *} \quad 25.25 \%$ \\
Sense of reciprocity (1-5) & & \\
Total & $-0.32(-0.51,-0.14)^{* *}$ & \\
Direct & $-0.25(-0.44,-0.06)^{* *}$ & \\
Indirect (detachment) & $-0.07(-0.11,-0.04)^{* * *}$ & $22.18 \%$ \\
Neighbourhood support (1-5) & & \\
Total & $-0.13(-0.29,0.04)$ & \\
Direct & $-0.09(-0.25,0.07)$ & \\
Indirect (detachment) & $-0.04(-0.06,-0.02)^{* * *}$ & - \\
Living with a partner & & \\
Total & $-0.27(-0.66,0.12)$ & \\
Direct & $-0.21(-0.60,0.18)$ & \\
Indirect (detachment) & $-0.06(-0.11,-0.02)^{* *}$ & - \\
\hline CI: & & \\
\hline
\end{tabular}

CI: confidence interval. All models were adjusted for sex, age, education level, malaise/pain, inability to perform daily activities, physical health problems, history of COVID-19 infection/quarantine and, if applicable, living with a partner or social support. Sampling weights were considered. The reference category for detachment is 'never' and for living with a partner is 'no'. Mediation effect is not provided when the total association was not significant

$* p<0.05 ; * * p<0.01 ; * * * p<0.001$

Likewise, emotional disorder symptoms mediated $36.2 \%$ of the relation between social support and STB. Among the social support components, the greatest mediation effect from emotional disorders was on social network size $(37.5 \%)$, followed by relations of reciprocity $(28.9 \%)$. In all of these associations the mediation effect of depressive symptoms was a little higher than that from anxiety symptoms.

Finally, Fig. 1 summarizes the mediation effects by detachment (Fig. 1a) and by emotional disorders symptoms (Fig. 1b) on STB.

\section{Discussion}

To the best of our knowledge, the present study is the first to analyse detachment as a factor related to available and real social support, and it confirms the complementary effect of detachment and social support in the pathways leading to
STB. It brings to light some under-investigated mechanisms regarding the role of detachment on these relationships, which could guide future interventions aimed at reducing STB prevalence in potentially traumatic events.

Our proposed hypothetical models of mediation on the association with STB were confirmed with high significance levels. On one hand, the relation between low social support and higher STB could be explained in part by the feeling of detachment and by emotional disorder symptoms. On the other hand, the relation between detachment and STB could partially be explained by emotional disorder symptoms, especially depressive symptoms. Our results are consistent with previous studies reporting that objective factors (e.g., social support and partner status) and subjective factors (e.g., loneliness and detachment) of social networks could have an independent effect on mental health, from which it follows that psychological and social interventions should be used in a complementary manner (Wang et al., 2018). In addition, other studies have found effects of moderation (Domènech-Abella et al., 2017) and mediation (Santini et al., 2020) of subjective factors on the association between objective factors of social networks and mental health.

Previous studies showed that the relationship between detachment and suicide ideation was stronger when comparing detachment to other PTSD symptoms (Davis et al., 2014). Moreover, low social support, as well as emotional disorders and remaining mental health problems, have been found to be robust risk factors for suicide (Too et al., 2019). Other researchers have focused on alternative explanatory constructs for STB related to social connectedness such as thwarted belongingness and perceived burdensomeness in the context of the interpersonal theory of suicide (Van Orden et al., 2010). Therefore, a merged and more complex model of the proposed relationships can be seen as a starting point for future research geared towards better and more detailed comprehension of the pathways leading from social connectedness to STB.

It has been suggested that examining these complex associations by stratifying the risk factors for suicidal ideation, plans, and attempts would provide improved understanding of how to create more specifically targeted interventions. In a case-control study of the contribution of schizoid personality disorder symptoms to suicide attempts (Levi-Belz et al., 2019), emotional detachment was found to be positively correlated with the medical lethality of the attempt, doubling the risk for more severe suicide attempts. This highlights the urgent necessity to better understand the effects of detachment on mental health in the general population, and the importance of also studying its relationship with protective factors, such as social support. In this regard, it would be of interest to do a deeper analysis of the general population regarding the relation of detachment and STB and related factors, distinguishing between the different types of STB and focussing on the lethality of the suicidal attempt. 
Table 4 Association (multivariate regression) of detachment and social network characteristics with STB, and emotional disorders symptoms (disentangled by depressive and anxiety symptoms) as mediating factors (KHB method)

\begin{tabular}{|c|c|c|c|}
\hline Detachment and social network characteristics & & Coefficient $(95 \% \mathrm{CI})$ & $\%$ Mediated \\
\hline \multicolumn{4}{|l|}{ Detachment (ref. never) } \\
\hline \multicolumn{4}{|l|}{ Sometimes } \\
\hline Total & & $0.87(0.40,1.34)^{* * *}$ & \\
\hline Direct & & $0.38(-0.12,0.88)$ & \\
\hline \multirow[t]{3}{*}{ Indirect } & Emotional disorders & $0.49(0.30,0.69)^{* * *}$ & $56.55 \%$ \\
\hline & (Depressive symptoms) & $0.28(0.13,0.43)$ & $32.34 \%$ \\
\hline & (Anxiety symptoms) & $0.21(0.05,0.37)$ & $24.21 \%$ \\
\hline \multicolumn{4}{|l|}{ Always } \\
\hline Total & & $2.27(1.73,2.82)^{* * *}$ & \\
\hline Direct & & $1.20(0.60,1.79)^{* * *}$ & \\
\hline \multirow[t]{3}{*}{ Indirect } & Emotional disorders & $1.08(0.78,1.37)^{* * *}$ & $47.31 \%$ \\
\hline & (Depressive symptoms) & $0.63(0.30,0.97)$ & $27.83 \%$ \\
\hline & (Anxiety symptoms) & $0.44(0.11,0.78)$ & $19.48 \%$ \\
\hline \multicolumn{4}{|l|}{ Social support (scale 3-14) } \\
\hline Total & & $-0.15(-0.25,-0.06)^{* *}$ & \\
\hline Direct & & $-0.10(-0.19,-0.00)^{*}$ & \\
\hline \multirow[t]{3}{*}{ Indirect } & Emotional disorders & $-0.06(-0.08,-0.04)^{* * *}$ & $36.15 \%$ \\
\hline & (Depressive symptoms) & $-0.03(-0.05,-0.01)$ & $21.54 \%$ \\
\hline & (Anxiety symptoms) & $-0.02(-0.04,-0.01)$ & $14.61 \%$ \\
\hline \multicolumn{4}{|l|}{ Social network size (1-4) } \\
\hline Total & & $-0.33(-0.58,-0.08)^{*}$ & \\
\hline Direct & & $-0.21(-0.46,0.05)$ & \\
\hline \multirow[t]{3}{*}{ Indirect } & Emotional disorders & $-0.12(-0.17,-0.07) * * *$ & $37.50 \%$ \\
\hline & (Depressive symptoms) & $-0.07(-0.12,-0.03)$ & $21.98 \%$ \\
\hline & (Anxiety symptoms) & $-0.05(-0.09,-0.01)$ & $15.53 \%$ \\
\hline \multicolumn{4}{|l|}{ Relations of reciprocity (1-5) } \\
\hline Total & & $-0.32(-0.51,-0.13)^{* *}$ & \\
\hline Direct & & $-0.23(-0.42,-0.04)^{*}$ & \\
\hline \multirow[t]{3}{*}{ Indirect } & Emotional disorders & $-0.09(-0.14,-0.05)^{* * *}$ & $28.91 \%$ \\
\hline & (Depressive symptoms) & $-0.06(-0.10,-0.02)$ & $19.09 \%$ \\
\hline & (Anxiety symptoms) & $-0.03(-0.06,-0.01)$ & $9.82 \%$ \\
\hline \multicolumn{4}{|l|}{ Neighbourhood support (1-5) } \\
\hline Total & & $-0.12(-0.28,0.04)$ & \\
\hline Direct & & $-0.06(-0.22,0.10)$ & \\
\hline \multirow[t]{3}{*}{ Indirect } & Emotional disorders & $-0.06(-0.09,-0.03)^{* * *}$ & - \\
\hline & (Depressive symptoms) & - & - \\
\hline & (Anxiety symptoms) & - & - \\
\hline \multicolumn{4}{|l|}{ Living with a partner (ref. no) } \\
\hline Total & & $-0.32(-0.73,-0.10)$ & \\
\hline Direct & & $-0.29(-0.71,-0.12)$ & \\
\hline \multirow[t]{3}{*}{ Indirect } & Emotional disorders & $-0.02(-0.09,0.04)$ & - \\
\hline & (Depressive symptoms) & - & - \\
\hline & (Anxiety symptoms) & - & - \\
\hline
\end{tabular}

CI: confidence interval. Ref: category of reference. All models were adjusted for sex, age, education level, malaise, inability to perform daily activities, physical health problems, history of COVID-19 infection/quarantine, and living with a partner. Sampling weights were considered. The variable 'living with a partner' was also adjusted for social support. $P$-values were not available with the disentangle option (i.e., for depressive and anxiety symptoms), so they were manually calculated. Mediation effect is not provided when the total association was not significant

$* p<0.05 ; * * p<0.01 ; * * * p<0.001$ 
High rates of STB, PTSD, emotional disorders, and other mental health conditions, together with their expected rise due to the COVID-19 pandemic, lay emphasis on the necessity of understanding the relationships between these constructs and potential points for psychological and social interventions. Our results are in line with Klomek's suggestion of focusing suicide prevention efforts on providing social support and online psychological help for dealing with the COVID-19 crisis (Klomek, 2020). These interventions should be designed considering both the objective social support measures and subjective perceptions of social connectedness. In accordance, policy makers and mental health experts need to call attention to the psychosocial effects of pandemics and implement promising interventions.

\section{Strengths and Limitations}

The results of the present study provide a richer understanding of the relationship among social support, detachment, emotional disorders symptoms, and STB. However, our findings should be interpreted considering some limitations. First, we used a cross-sectional design, which limits conclusions that may be drawn regarding causality. Future longitudinal studies should be designed to overcome this limitation. Secondly, the use of questionnaires and self-reported data can be tied to response bias which may affect the validity of the research. In this study, the most probable bias was social desirability, which was overcome by using clinically validated screening instruments to asses all the possible variables. Thirdly, some covariates related with the participants such as their lifestyle, traumatic events related with the pandemic, time spent on COVID-19 response activities, or other factors related to health behaviours that may be potential confounders could not be considered in this study. However, we have used several control variables about the participants' physical and mental health, as well as regarding their sociodemographic and socioeconomic characteristics, in order to minimize the bias. Finally, detachment has been relatively understudied, so the scarce literature on the topic hinders contrasting of our findings with others. Despite the aforementioned limitations, this study offers pioneering knowledge on the effects of detachment on STB in the context of the COVID19 lockdown. Among its strengths are its representativeness of the general adult population and the use of validated measures and adjustments for a wide range of covariates, conferring high reliability on the results obtained.

\section{Conclusions}

The present findings suggest that social support and detachment might be renewed and effective points of treatment interventions to reduce symptoms of emotional disorders and
STB among the general population, especially in a traumatic and long-lasting context such as the COVID-19 pandemic. However, as these findings are derived from cross-sectional data, additional longitudinal data and data from different settings are still needed to confirm these associations and to test more complex models that might allow for the observation of the reciprocal effects among all the considered variables. Community-level interventions and prevention efforts focused on the appearance of detachment during the pandemic are essential to prevent a further increase in STB cases. Nevertheless, these actions should also address the changes in social networks, given the importance of social support and its components in preventing STB and emotional disorders.

Acknowledgments This work was supported by the Instituto de Salud Carlos III, Ministerio de Ciencia, e Innovación, [grant number COV20/ 00711]; and the RESPOND project funded by the European Union's Horizon 2020 Framework Programme [grant number 101016127]. AGP work is supported by the Secretariat of Universities and Research of the Generalitat de Catalunya and the European Social Fund (2021 FI B 00839). JD-A has a "Juan de la Cierva" research contract awarded by the Ministry of Science and Innovation, Government of Spain (FJC2019038955-I). We thank Thomas Yohannan for the help in English language editing. Finally, we would like to express special gratitude to all the participants for their generous contribution which made this work possible.

Authors' Contributions The study design was planned by AG-P, MF-N, PC-N, PM, GV, BO, JA, JMH, and JD-A. AG-P conducted the data analyses. JD-A and AG-P drafted the article. JMH supervised the data analyses and development of the paper. The paper was edited and reviewed by all the authors. All authors read and approved the final manuscript.

Funding This study was supported by the Instituto de Salud Carlos III, Ministerio de Ciencia, e Innovación, [grant number COV20/00711]; and the RESPOND project funded by the European Union's Horizon 2020 Framework Programme [grant number 101016127]. AG-P work is supported by the Secretariat of Universities and Research of the Generalitat de Catalunya and the European Social Fund (2021 FI B 00839). JD-A has a "Juan de la Cierva" research contract awarded by the Ministry of Science and Innovation, Government of Spain (FJC2019-038955-I).

Availability of Data and Material The de-identified participant data as well as the study protocol, statistical analysis plan, and data dictionaries used for this study are available as from publication and upon reasonable request from the corresponding author (AG-P; aina.gabarrell@sjd.es) as long as the main objective of the data sharing request is replicating the analysis and findings as reported in this paper (without investigator support), after approval of a proposal, and with a signed data access agreement.

Code Availability Not applicable.

\section{Declarations}

Ethics Approval Ethical approval from the relevant ethical committees (Parc Sanitari Sant Joan de Déu, Barcelona, Spain (PIC 86-20) and Parc de Salut Mar Clinical Research Ethics Committee (protocol 2020/9203/I)) was obtained. 
Consent to Participate Participants were thoroughly informed about the objectives and procedures of the study. Each respondent had to provide oral consent before participation.

Consent for Publication Participants were fully informed and provided consent regarding publications derived from the study.

Conflict of Interest The authors declare that they have no conflict of interest with respect to the research, authorship, and/or publication of this article.

\section{References}

Alfonso-Sánchez, J. L., Martin-Moreno, J. M., Martinez, I. M., \& Martinez, A. A. (2020). Epidemiological study and cost analysis of suicide in Spain: Over 100 years of evolution. Archives of Suicide Research, 24(sup2), S356-S369. https://doi.org/10.1080/ 13811118.2019.1612802

American Psychiatric Association. (2013). Diagnostic and statistical manual of mental disorders DSM-5 fifth edition. Author.

Banerjee, D., Kosagisharaf, J. R., \& Sathyanarayana Rao, T. S. (2021). 'The dual pandemic' of suicide and COVID-19: A biopsychosocial narrative of risks and prevention. Psychiatry Research, 295, 113577. https://doi.org/10.1016/j.psychres.2020.113577

Breen, R., Karlson, K. B., \& Holm, A. (2013). Total, direct, and indirect effects in logit and probit models. Sociological Methods \& Research, 42(2), 164-191. https://doi.org/10.1177/ 0049124113494572

Brooks, S. K., Webster, R. K., Smith, L. E., Woodland, L., Wessely, S., Greenberg, N., \& Rubin, G. J. (2020). The psychological impact of quarantine and how to reduce. The Lancet, 395, 912-920

Bryant-Genevier, J., Rao, C. Y., Lopes-Cardozo, B., Kone, A., Rose, C., Thomas, I., et al. (2021). Symptoms of depression, anxiety, posttraumatic stress disorder, and suicidal ideation among state, tribal, local, and territorial public health workers during the COVID-19 pandemic - United States, March-April 2021. MMWR. Morbidity and Mortality Weekly Report, 70(26), 947-952. https:// doi.org/10.15585/mmwr.mm7026e1

Calati, R., Ferrari, C., Brittner, M., Oasi, O., Olié, E., Carvalho, A. F., \& Courtet, P. (2019). Suicidal thoughts and behaviors and social isolation: A narrative review of the literature. Journal of Affective Disorders, 245, 653-667. https://doi.org/10.1016/j.jad.2018.11.022

Chen, P., Pusica, Y., Sohaei, D., Prassas, I., \& Diamandis, E. (2021). An overview of mental health during the COVID-19 pandemic. Diagnosis. https://doi.org/10.1515/dx-2021-0046

Davis, M. T., Witte, T. K., \& Weathers, F. W. (2014). Posttraumatic stress disorder and suicidal ideation: The role of specific symptoms within the framework of the interpersonal-psychological theory of suicide. Psychological Trauma Theory Research Practice and Policy, 6(6), 610-618. https://doi.org/10.1037/a0033941

Domènech-Abella, J., Lara, E., Rubio-Valera, M., Olaya, B., Moneta, M. V., Rico-Uribe, L. A., Ayuso-Mateos, J. L., Mundó, J., \& Haro, J. M. (2017). Loneliness and depression in the elderly: The role of social network. Social Psychiatry and Psychiatric Epidemiology, 52(4), 381-390. https://doi.org/10.1007/s00127-017-1339-3

Durkheim, É. (1951). Suicide: A study in sociology (J. A. Spaulding \& G. Simpson, Trans.). Free Press (Original work published 1897). https://doi.org/10.4324/9780203994320

Ettman, C. K., Abdalla, S. M., Cohen, G. H., Sampson, L., Vivier, P. M., \& Galea, S. (2020). Prevalence of depression symptoms in US adults before and during the COVID-19 pandemic. JAMA Network Open, 3(9), e2019686. https://doi.org/10.1001/jamanetworkopen. 2020.19686
Eurostat. (2021). Database - Eurostat. https://ec.europa.eu/eurostat/data/ database. Accessed 5 February 2021

Gunnell, D., Appleby, L., Arensman, E., Hawton, K., John, A., Kapur, N., Khan, M., O'Connor, R. C., Pirkis, J., Appleby, L., Arensman, E., Caine, E. D., Chan, L. F., Chang, S. S., Chen, Y. Y., Christensen, H., Dandona, R., Eddleston, M., Erlangsen, A., et al. (2020). Suicide risk and prevention during the COVID-19 pandemic. The Lancet Psychiatry, 7(6), 468-471. https://doi.org/10.1016/S22150366(20)30171-1

Hawton, K., Casañas, I., Comabella, C., Haw, C., \& Saunders, K. (2013). Risk factors for suicide in individuals with depression: A systematic review. Journal of Affective Disorders, 147, 17-28. https://doi.org/ 10.1016/j.jad.2013.01.004

Hyatt, C. S., Maples-Keller, J. L., Crowe, M. L., Sleep, C. E., Carter, S. T., Michopoulos, V., Stevens, J. S., Jovanovic, T., Bradley, B., Miller, J. D., \& Powers, A. (2020). Psychometric properties of the personality inventory for DSM-5-brief form in a community sample with high rates of trauma exposure. Journal of Personality Assessment, 103, 1-10. https://doi.org/10.1080/00223891.2020. 1713138

INE. (2020). España en cifras 2020; Instituto Nacional de Estadística. ISSN 2255-0410. https://www.ine.es/ss/Satellite?L=es_ES\&c= INEPublicacion_C\&cid=1259924856416\&p= $1254735110672 \&$ pagename $=$ Productos YServicios $\%$ 2FPYSLayout\&param1=PYSDetalleGratuitas. Accessed 20 December 2020

John, A., Pirkis, J., Gunnell, D., Appleby, L., \& Morrissey, J. (2020, November 12). Trends in suicide during the covid-19 pandemic. The BMJ. BMJ Publishing Group. https://doi.org/10.1136/bmj. $\mathrm{m} 4352$

Kessler, R. C., \& Üstün, B. B. (2004). The World Mental Health (WMH) survey initiative version of the World Health Organization (WHO) composite international diagnostic interview (CIDI). International Journal of Methods in Psychiatric Research, 13(2), 93-117. https:// doi.org/10.1002/mpr.168

Klomek, A. B. (2020). Suicide prevention during the COVID-19 outbreak. The Lancet Psychiatry, 7, 390. https://doi.org/10.1016/ S2215-0366(20)30142-5

Kocalevent, R. D., Berg, L., Beutel, M. E., Hinz, A., Zenger, M., Härter, M., Nater, U., \& Brähler, E. (2018). Social support in the general population: Standardization of the Oslo social support scale (OSSS3). BMC Psychology, 6(1), 4-11. https://doi.org/10.1186/s40359018-0249-9

Kroenke, K., Strine, T. W., Spitzer, R. L., Williams, J. B. W., Berry, J. T., \& Mokdad, A. H. (2009). The PHQ-8 as a measure of current depression in $\mathrm{t} 0068 \mathrm{e}$ general population. Journal of Affective Disorders, 114(1-3), 163-173. https://doi.org/10.1016/j.jad.2008. 06.026

Leaune, E., Samuel, M., Oh, H., Poulet, E., \& Brunelin, J. (2020). Suicidal behaviors and ideation during emerging viral disease outbreaks before the COVID-19 pandemic: A systematic rapid review. Preventive Medicine, 141, 106264. https://doi.org/10.1016/j.ypmed. 2020.106264

Levi-Belz, Y., Gvion, Y., Levi, U., \& Apter, A. (2019). Beyond the mental pain: A case-control study on the contribution of schizoid personality disorder symptoms to medically serious suicide attempts. Comprehensive Psychiatry, 90, 102-109. https://doi.org/ 10.1016/j.comppsych.2019.02.005

Li, J., Yang, Z., Qiu, H., Wang, Y., Jian, L., Ji, J., \& Li, K. (2020). Anxiety and depression among general population in China at the peak of the COVID-19 epidemic. World Psychiatry, 19(2), 249250. https://doi.org/10.1002/wps.20758

Liu, C. H., Zhang, E., Wong, G. T. F., Hyun, S., \& Hahm, H. “. C.”. (2020). Factors associated with depression, anxiety, and PTSD symptomatology during the COVID-19 pandemic: Clinical 
implications for U.S. young adult mental health. Psychiatry Research, 290, 113172. https://doi.org/10.1016/j.psychres.2020. 113172

Lorant, V., De Gelder, R., Kapadia, D., Borrell, C., Kalediene, R., Kovács, K., et al. (2018). Socioeconomic inequalities in suicide in Europe: The widening gap. British Journal of Psychiatry, 212(6), 356-361. https://doi.org/10.1192/bjp.2017.32

Mazza, C., Ricci, E., Biondi, S., Colasanti, M., Ferracuti, S., Napoli, C., \& Roma, P. (2020a). A nationwide survey of psychological distress among italian people during the covid-19 pandemic: Immediate psychological responses and associated factors. International Journal of Environmental Research and Public Health, 17(9), 114. https://doi.org/10.3390/ijerph17093165

Mazza, M. G., De Lorenzo, R., Conte, C., Poletti, S., Vai, B., Bollettini, I., et al. (2020b). Anxiety and depression in COVID-19 survivors: Role of inflammatory and clinical predictors. Brain, Behavior, and Immunity, 89, 594-600. https://doi.org/10.1016/j.bbi.2020.07.037

Mickey, R. M., \& Greenland, S. (1989). The impact of confounder selection criteria on effect estimation. American Journal of Epidemiology, 129(1), 125-137. https://doi.org/10.1093/ oxfordjournals.aje.a115101

MIND/COVID-19: Mental health Impact and NeeDs associated with COVID-19: A comprehensive national evaluation in Spain (COV20/00711). (2020). Retrieved from http://eu-isciii.es/ covidfundinspain/mind-covid-19-mental-health-impact-and-needsassociated-with-covid-19-a-comprehensive-nationalevaluation-inspain/. Accessed 20 December 2020

Miret, M., Caballero, F. F., Huerta-Ramírez, R., Moneta, M. V., Olaya, B., Chatterji, S., Haro, J. M., \& Ayuso-Mateos, J. L. (2014). Factors associated with suicidal ideation and attempts in Spain for different age groups. Prevalence before and after the onset of the economic crisis. Journal of Affective Disorders, 163, 1-9. https://doi.org/10. 1016/j.jad.2014.03.045

Newman, M. G., Zuellig, A. R., Kachin, K. E., Constantino, M. J., Przeworski, A., Erickson, T., \& Cashman-McGrath, L. (2002). Preliminary reliability and validity of the generalized anxiety disorder questionnaire-IV: A revised self-report diagnostic measure of generalized anxiety disorder. Behavior Therapy, 33(2), 215-233. https://doi.org/10.1016/S0005-7894(02)80026-0

Posner, K., Brown, G. K., Stanley, B., Brent, D. A., Yershova, K. V., Oquendo, M. A., Currier, G. W., Melvin, G. A., Greenhill, L., Shen, S., \& Mann, J. J. (2011). The Columbia-suicide severity rating scale: Initial validity and internal consistency findings from three multisite studies with adolescents and adults. American Journal of Psychiatry, 168(12), 1266-1277. https://doi.org/10.1176/appi.ajp. 2011.10111704

Ritchie, H., Ortiz-Ospina, E., Beltekian, D., Mathieu, E., Hasell, J., Macdonald, B., et al. (2020, March 5). Coronavirus pandemic (COVID-19). Our World in Data. https://ourworldindata.org/ coronavirus. Accessed 12 July 2021

Santini, Z. I., Koyanagi, A., Tyrovolas, S., Mason, C., \& Haro, J. M. (2015). The association between social relationships and depression: A systematic review. Journal of Affective Disorders, 175, 53-65. https://doi.org/10.1016/j.jad.2014.12.049

Santini, Z. I., Jose, P. E., York Cornwell, E., Koyanagi, A., Nielsen, L., Hinrichsen, C., Meilstrup, C., Madsen, K. R., \& Koushede, V. (2020). Social disconnectedness, perceived isolation, and symptoms of depression and anxiety among older Americans (NSHAP): A longitudinal mediation analysis. The Lancet Public Health, 5(1), e62-e70. https://doi.org/10.1016/S2468-2667(19)30230-0

Sher, L. (2020a). The impact of the COVID-19 pandemic on suicide rates. QJM : Monthly Journal of the Association of Physicians, 113, 707-712. https://doi.org/10.1093/qjmed/hcaa202

Sher, L. (2020b). Are COVID-19 survivors at increased risk for suicide? Acta Neuropsychiatrica, 32, 270. https://doi.org/10.1017/neu.2020. 21

Stuckler, D., Basu, S., Suhrcke, M., Coutts, A., \& McKee, M. (2009). The public health effect of economic crises and alternative policy responses in Europe: An empirical analysis. The Lancet, 374(9686), 315-323. https://doi.org/10.1016/S0140-6736(09)61124-7

Tanaka, T., \& Okamoto, S. (2021). Increase in suicide following an initial decline during the COVID-19 pandemic in Japan. Nature Human Behaviour, 5, 1-10. https://doi.org/10.1038/s41562-020-01042-z

Thakur, V., \& Jain, A. (2020). COVID 2019-suicides: A global psychological pandemic. Brain, Behavior, and Immunity, 88, 952-953. https://doi.org/10.1016/j.bbi.2020.04.062

Too, L. S., Spittal, M. J., Bugeja, L., Reifels, L., Butterworth, P., \& Pirkis, J. (2019). The association between mental disorders and suicide: A systematic review and meta-analysis of record linkage studies. Journal of Affective Disorders. https://doi.org/10.1016/j.jad.2019. 08.054

van Buuren, S. (2018). Flexible imputation of missing data, second edition. Flexible imputation of missing data, second edition. Chapman and Hall/CRC. https://doi.org/10.1201/9780429492259

Van Orden, K. A., Witte, T. K., Cukrowicz, K. C., Braithwaite, S., Selby, E. A., \& Joiner, T. E. (2010). The interpersonal theory of suicide. Psychological Review, 117(2), 575-600. https://doi.org/10.1037/ a0018697

Vindegaard, N., \& Benros, M. E. (2020). COVID-19 pandemic and mental health consequences: Systematic review of the current evidence. Brain, Behavior, and Immunity, 89, 531-542. https://doi.org/10. 1016/j.bbi.2020.05.048

Wang, J., Mann, F., Lloyd-Evans, B., Ma, R., \& Johnson, S. (2018). Associations between loneliness and perceived social support and outcomes of mental health problems: A systematic review. BMC Psychiatry, 18(1), 156. https://doi.org/10.1186/s12888-018-1736-5

WHO. (2019). Suicide. https://www.who.int/health-topics/suicide\#tab= tab_1. Accessed 20 December 2020

WHO. (2020). WHO coronavirus disease (COVID-19) dashboard. https://covid19.who.int/. Accessed 5 February 2021

Xiong, J., Lipsitz, O., Nasri, F., Lui, L. M. W., Gill, H., Phan, L., ChenLi, D., Iacobucci, M., Ho, R., Majeed, A., \& McIntyre, R. S. (2020). Impact of COVID-19 pandemic on mental health in the general population: A systematic review. Journal of Affective Disorders, 277(June), 55-64. https://doi.org/10.1016/j.jad.2020.08.001

Yousefi, B., Valizadeh, S., Ghaffari, H., Vahedi, A., Karbalaei, M., \& Eslami, M. (2020). A global treatments for coronaviruses including COVID-19. Journal of Cellular Physiology, 235(12), 9133-9142. https://doi.org/10.1002/jcp.29785

Publisher's Note Springer Nature remains neutral with regard to jurisdictional claims in published maps and institutional affiliations. 